\title{
ENFERMIDADES DIAGNOSTICADAS NA GENITÁLIA EXTERNA DE TOUROS: ESTUDO RETROSPECTIVO (2007 - 2013)
}

\section{DISEASES DIAGNOSED IN THE EXTERN GENITAL ORGANS OF BULLS: RETROSPECTIVE STUDY (2007-2013)}

\author{
Rogério Elias Rabelo ${ }^{1}$ \\ Luiz Antônio Franco da Silva² \\ Valcinir Aloísio Scalla Vulcani ${ }^{1}$ \\ Fabiano José Ferreira de Sant'Ana ${ }^{3}$ \\ Bruno Moraes Assis ${ }^{4}$ \\ Andressa Sabine Rabbers ${ }^{4}$ \\ 1Professores Doutores da Universidade Federal de Goiás, Jataí, GO, Brasil - rabelovet@yahoo.com.br \\ 2Professor Doutor da Universidade Federal de Goiás, Goiânia, GO, Brasil \\ ${ }^{3}$ Professor Doutor da Universidade de Brasília, Brasília, DF, Brasil \\ 4Pós-graduandos da Universidade Federal de Goiás, Goiânia, GO, Brasil
}

\begin{abstract}
Resumo:
Apesar da importância de se conhecerem os dados epidemiológicos sobre as enfermidades que acometem a genitália externa de touros, poucas investigações têm sido conduzidas sobre o tema. Este trabalho objetiva realizar um estudo epidemiológico de enfermidades diagnosticadas na genitália externa de touros, em 215 propriedades rurais distribuídas nas cinco mesorregiões do Estado de Goiás, Brasil, entre os anos de 2007 e 2013. Pôde-se notar que o maior número de casos foi verificado na mesorregião do sudoeste goiano (64\%), seguida do noroeste de Goiás (18,5\%). Verificou-se que, dos touros afetados, $156(71,89 \%)$ eram zebuínos, 47 (21,66\%) taurinos e 14 (6,45\%) mestiços. Dentre as enfermidades, a acropostite-fimose se destacou, seguida do fibropapiloma de glande, abscesso prepucial e fratura peniana. Confirmaram-se como fatores de risco, aspectos multifatoriais, com destaque para a condição morfológicas dos animais, ambiente, manejo e os traumas. Em muitos casos, a ausência ou o número insuficiente de médicos veterinários capacitados que atuam no campo foi mencionado como um dos principais pontos de estrangulamento para a realização do diagnóstico, tratamento e prevenção das enfermidades, o que evitaria o descarte prematuro dos animais.
\end{abstract}

Palavras-chave: Doenças de bovinos; epidemiologia; sistema genital masculino.

\begin{abstract}
:
Despite the importance of knowing epidemiological data on diseases that affect the external genitalia of bulls, few investigations have been conducted on the topic. This work aimed to conduct an epidemiological study of diseases diagnosed in the external genitalia of bulls in 215 farms distributed in the five mesoregions of the state of Goiás, Brazil, between 2007 and 2013. We verified that the largest number of cases were observed at southwestern region (64\%), followed by the northwestern region $(18.5 \%)$. We found that of the affected bulls, 156 (71.89\%) were Zebu, 47 (21.66\%) taurine and $14(6.45 \%)$ were mixed breeds. Among the diseases, acropostite-phimosis was the more frequent,
\end{abstract}


followed by fibropapiloma of the glans, prepuce abscess and penile fracture. The main risk factors were multifactorial aspects, including the morphological condition of the animals, environment, management and traumas. In many cases, the absence or insufficient number of trained veterinarians who work on the field was mentioned as one of the major bottlenecks for the realization of the diagnosis, treatment and prevention of diseases, preventing premature disposal of the animals.

Keywords: Diseases of cattle; epidemiology; male genital system.

Recebido em: 08 jul. 2014

Aceito em: 21 nov. 2014

\section{Introdução}

Diversas enfermidades da genitália externa do touro podem interferir em sua eficiência reprodutiva, podendo dificultar ou mesmo inviabilizar o coito, determinando prejuízos ao criatório. Alguns aspectos morfológicos e fisiopatológicos do sistema genital, especialmente prepúcio e folheto prepucial interno pendulosos, orifício prepucial largo, bem como agenesia ou lesões nos músculos prepucias são considerados fatores predisponentes na etiopatogenia dessas afecções ${ }^{(1-4)}$. A etiologia de algumas injúrias que acometem o aparelho reprodutor do touro, especialmente as traumáticas, foram previamente estudadas. Características raciais e práticas de manejo são fatores que podem contribuir de sobremaneira para o desenvolvimento dessas desordens ${ }^{(5)}$. Entretanto, algumas informações epidemiológicas ainda não foram totalmente elucidadas, necessitando de pesquisas científicas mais aprofundadas sobre o assunto.

Apesar da importância dos dados epidemiológicos para melhor conhecimento e controle das diferentes enfermidades responsáveis pelas injúrias que acometem a genitália externa dos touros, poucas investigações têm sido conduzidas sobre o tema. Estudo retrospectivo realizado durante os anos de 1982 a 2007, em um total de 12.320 touros constatou que raças zebuínas apresentam maior prevalência das enfermidades que acometem a genitália externa, com destaque para a acropostitefimose, e que os aspectos morfológicos, informações epidemiológicas e sistema de manejo adotado nas propriedades são considerados pontos importantes a serem avaliados ${ }^{(6)}$.

Este trabalho realizou um estudo epidemiológico de enfermidades diagnosticadas na genitália externa de touros, em 215 propriedades rurais no estado de Goiás, Brasil, entre os anos de 2007 e 2013.

\section{Material e Métodos}

O estudo foi realizado entre fevereiro de 2007 e dezembro de 2013, em 215 propriedades rurais localizadas nas cinco mesorregiões do Estado de Goiás. As propriedades foram selecionadas com base no critério de existência de reprodutores bovinos portadores de enfermidades na genitália externa, resultando em dificuldade, inabilidade na cópula ou perda de libido.

Todas as visitas foram realizadas após solicitação do proprietário, tendo como queixa principal algum distúrbio que acometia o aparelho reprodutor de um ou mais touros, com consequente desencadeamento de incapacidade de realização da cópula, contribuindo para redução da fertilidade no plantel. Na propriedade de origem, os animais foram submetidos a exame clínico geral e específico da genitália, alguns necessitando de anestesia locorregional para dessensibilização dos nervos 
pudendos e hemorroidais $^{(7)}$, visando exposição do pênis para permitir a elucidação da enfermidade. Quando não foi possível identificar a enfermidade por meio dos métodos semiotécnicos de rotina, utilizou-se o teste de capacidade de serviço, preconizado por Santos et al. ${ }^{(8)}$, para confirmar a suspeita clínica. Esse teste fundamentou-se na observação do comportamento sexual do touro diante de uma fêmea bovina em cio natural ou induzido terapeuticamente ${ }^{(9)}$.

Os touros foram avaliados individualmente por dez minutos, em curral fechado, verificando-se dentre outros fatores, a exposição e correto direcionamento do pênis por ocasião da monta, lesões teciduais penianas e peri-penianas, libido e efetivação da cópula. Os casos de enfermidades identificados foram tratados, quando possível, cirurgicamente, seguindo metodologias propostas pela literatura ${ }^{(4,10,11,13)}$. Quando não havia possibilidade ou indicação para a intervenção cirúrgica, tendo em vista o valor intrínseco do touro ou a gravidade da lesão, recomendou-se o descarte.

Paralelamente, um questionário elaborado com base em Thrusfield et al. ${ }^{(13)}$ foi aplicado em todas as propriedades e respondido pelo proprietário, responsável técnico ou gerente. Dentre os questionamentos levantados, enfatizaram-se dados relacionados à raça e idade dos bovinos acometidos, época do ano de maior ocorrência, variedade e tipo de pastagens, manejo reprodutivo adotado, etiopatogenia da enfermidade, medidas de controle, tratamentos adotados, impacto econômico, destino dos animais enfermos e existência ou não, na região, de profissionais especializados para resolução dos problemas.

Em alguns casos, amostras dos órgãos reprodutivos afetados foram coletadas no momento da cirurgia para avaliação histopatológica com fins diagnósticos. Os fragmentos foram fixados em formol 10\% e processados rotineiramente para coloração com hematoxilina-eosina (HE).

Para análise dos dados obtidos empregou-se estatística descritiva apresentadas por meio de tabelas e figuras $^{(14)}$.

\section{Resultados}

De um total de 3.125 touros presentes nas propriedades do estudo, $217(14,4 \%)$ animais de diferentes raças, com idade entre 25 e 120 meses, apresentaram alguma enfermidade da genitália externa passível de tratamento cirúrgico e que dificultava ou mesmo incapacitava o bovino para reprodução. Verificou-se que, dos touros enfermos, 156 (71,89\%) eram zebuínos, 47 (21,66\%) taurinos e 14 (6,45\%) mestiços (Bos indicus indicus x Bos taurus taurus). Dos 156 touros zebuínos, a maior frequência foi observada na raça Nelore, com 105 (67,30\%) animais portadores de lesões na genitália externa, seguida de 20,51\% nas raças Gir (32/156), 8,35\% Tabapuã (13/156), 2,56\% Guzerá (4/156), 0,64\% Indubrasil (1/156) e 0,64\% Curraleiro (1/156). Já nos animais taurinos, detectou-se que, dentre as raças com maior frequência de enfermidades, destacaram-se a Holandesa com $28(59,57 \%)$ casos, 19,15\% Simental (9/47), 8,51\% Pardo Suíço (4/47), 6,38\% Limousin (3/47), 2,13\% Senepol (1/47), 2,13\% Blonde d'Aquitaine (1/47) e 2,13\% Aberdeen Angus (1/47). Das raças mestiças (Bos indicus indicus x Bos taurus taurus), verificou-se uma maior frequência das enfermidades na raça Girolando, com $11(78,57 \%)$ touros acometidos, conforme descrito na Tabela 1.

A Figura 1 apresenta o percentual de atendimentos realizados por ocasião do estudo nas diferentes mesorregiões do estado de Goiás. Observou-se que mais da metade dos casos foi diagnosticada na região Sul do Estado.

A Tabela 2 evidencia o quantitativo de propriedades atendidas em diferentes municípios, de acordo com cada mesorregião selecionadas para o estudo. 
Tabela 1: Frequência das raças de 217 touros portadores de enfermidades da genitália externa, em 215 propriedades rurais do Estado de Goiás, no período de 2007 a 2013

\begin{tabular}{cccc}
\hline Subespécies & Raças & Quantidade & Frequência (\%) \\
\hline & Nelore & 105 & 67,30 \\
& Gir & 32 & 20,51 \\
& Tabapuã & 13 & 8,35 \\
Bos taurus indicus & Guzerá & 04 & 2,56 \\
& Indubrasil & 01 & 0,64 \\
& Curraleiro & 01 & 0,64 \\
& Subtotal & $\mathbf{1 5 6}$ & $\mathbf{1 0 0}$ \\
& & & \\
& Holandês & 28 & 59,57 \\
Bos taurus taurus & Simental & 09 & 19,15 \\
& Pardo Suíço & 04 & 8,51 \\
& Limousin & 03 & 6,38 \\
& Senepol & 01 & 2,13 \\
& Blonde d'Aquitaina & 01 & 2,13 \\
& Aberdeen Angus & 01 & 2,13 \\
& Subtotal & $\mathbf{4 7}$ & $\mathbf{1 0 0}$ \\
& & & 78,57 \\
Mestiços & Girolando & 11 & 21,43 \\
& Outras & 03 & $\mathbf{1 0 0}$ \\
\hline Total Geral & Subtotal & $\mathbf{1 4}$ & \\
\hline
\end{tabular}

\section{ESTADO DE GOIÁS - MESORREGIOES}

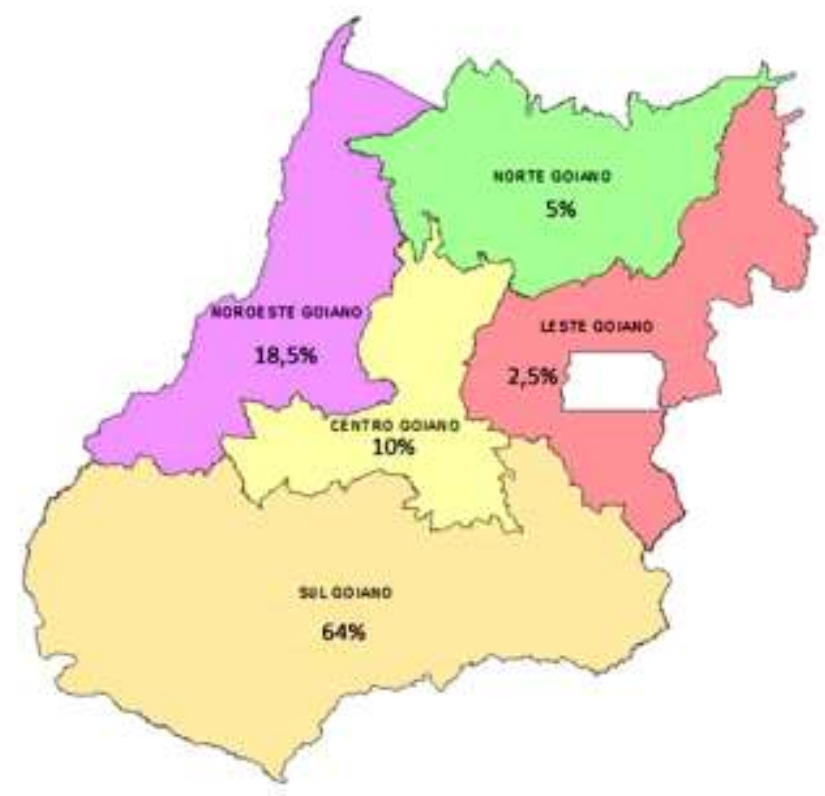

Figura 1: Mapa do Estado de Goiás mostrando o percentual de enfermidades da genitália externa de touros por cada mesorregião avaliada, entre 2007 e 2013. 
Tabela 2. Distribuição dos municípios e mesorregiões do Estado de Goiás onde foram diagnosticadas enfermidades da genitália externa de touros entre 2007 e 2013

\begin{tabular}{|c|c|}
\hline Municípios/mesorregiões de Goiás & Número de propriedades atendidas \\
\hline \multicolumn{2}{|l|}{ Norte } \\
\hline Mozarlândia & 03 \\
\hline Nova Crixás & 04 \\
\hline São Miguel do Araguaia & 03 \\
\hline $\begin{array}{l}\text { Sub total } \\
\text { Noroeste }\end{array}$ & 10 \\
\hline Araguapaz & 03 \\
\hline Britânia & 04 \\
\hline Caiapônia & 15 \\
\hline Iporá & 08 \\
\hline Jussara & 04 \\
\hline São Luiz dos Montes Belos & 05 \\
\hline Sub total & 39 \\
\hline \multicolumn{2}{|l|}{ Leste } \\
\hline Luziânia & 01 \\
\hline Padre Bernardo & 04 \\
\hline Sub total & 05 \\
\hline $\begin{array}{c}\text { Centro } \\
\text { Anápolis }\end{array}$ & 02 \\
\hline Bela Vista de Goiás & 01 \\
\hline Ceres & 01 \\
\hline Goiânia & 05 \\
\hline Goianira & 01 \\
\hline Guapó & 01 \\
\hline Inhumas & 02 \\
\hline Jaraguá & 05 \\
\hline Teresópolis & 02 \\
\hline Sub total & 20 \\
\hline \multicolumn{2}{|l|}{ Sul } \\
\hline Aporé & 03 \\
\hline Cachoeira Alta & 02 \\
\hline Catalão & 05 \\
\hline Indiara & 04 \\
\hline Itumbiara & 01 \\
\hline Jataí & 46 \\
\hline Mineiros & 08 \\
\hline Morrinhos & 07 \\
\hline Orizona & 04 \\
\hline Pires do Rio & 01 \\
\hline Pontalina & 08 \\
\hline Portelândia & 08 \\
\hline Quirinópolis & 04 \\
\hline Rio Verde & 23 \\
\hline Serranópolis & 09 \\
\hline Vicentinópolis & 08 \\
\hline Sub total & 141 \\
\hline TOTAL GERAL & 215 \\
\hline
\end{tabular}


Por ocasião das visitas às propriedades e avaliação dos questionários, verificou-se 87,9\% (189/215) das fazendas adotavam o manejo de monta natural, com ou sem estação de monta, porém, sem a adoção do sistema de monta controlada. Nestas, o pastejo era efetuado geralmente em pastagens mal manejadas, degradadas e com excesso de ervas daninhas, como lobeira (Solanum lycocarpum), arranha-gato (Acacia plumosa), malícia (Mimosa sensitiva), dentre outras, ou mesmo em pastos nativos. Relatou-se também a ação espoliativa por ectoparasitas e traumatismos promovidos por bicadas de aves domésticas e silvestres. As demais propriedades (26; 12,10\%) adotavam biotecnologias no manejo reprodutivo como a inseminação artificial (IA), inseminação artificial em tempo fixo (IATF), transferência de embriões (TE), sendo touros empregados somente para repasse de vacas. Nessas propriedades, o pastejo era efetuado em piquetes limpos e bem manejados.

Quanto à idade, observou-se que 84,33\% (183/217) dos touros afetados tinham idade igual ou superior a 60 meses, 12,44\% (27/217) entre 40 a 60 e 3,23\% (7/217) inferior a 40 meses. Independente da enfermidade genital diagnosticada, pôde-se também verificar que a maior ocorrência se deu ao término da estação de monta, quando esta era adotada. Do total de animais do estudo, 169 (78\%) foram acometidos por enfermidades prepuciais, 41 (19\%) por lesões penianas e sete $(3,0 \%)$ por alterações da bolsa escrotal ou testículos. Dentre as principais enfermidades prepuciais, a acropostitefimose foi diagnosticada em 136 touros, correspondendo a 80,49\% das lesões nessa região anatômica, seguida de $12(7,10 \%)$ casos de abscesso prepucial, nove $(5,32 \%)$ de fimose, cinco $(2,96)$ de frênulo persistente, três $(1,77 \%)$ de dilaceração prepucial e divertículo prepucial posterior e um $(0,59 \%)$ de divertículo prepucial anterior. Quanto às enfermidades que acometeram a região peniana, foram diagnosticados $13(31,70 \%)$ casos de fibropapiloma de glande, $11(26,83 \%)$ de fratura, nove $(21,95 \%)$ de desvio prematuro, quatro de parafimose $(9,76 \%)$ e quatro de urolitíase $(9,76 \%)$. Das sete injúrias diagnosticadas na bolsa escrotal e testículos verificou-se três $(42,85 \%)$ casos de orquite, dois $(28,57 \%)$ de hérnia inguino-escrotal, um $(14,29 \%)$ de abscesso escrotal e um $(14,29 \%)$ de seminoma (Tabela 3).

Dos 136 touros com diagnóstico de acropostite-fimose, seis raças destacaram-se em função do número de animais acometidos, sendo 90 (66,20\%) da raça Nelore, 20 (14,70\%) Gir, nove (6,62\%) Tabapuã, oito (5,88\%) Girolando, três (2,20\%) Holandês, três (2,20\%) Guzerá, dois (1,47\%) Simental e um $(0,73 \%)$ Indubrasil. Dessa forma, os zebuínos representaram 123 (90,44\% do total) dos bovinos afetados por essa condição. Nos casos de fibropapiloma peniano, os entrevistados responderam que outros bovinos da fazenda apresentavam lesões semelhantes na pele.

Dos 12 casos de abscessos prepuciais, referentes a 5,53\% do total de lesões observadas, nove (75\%) foram evidenciados em touros taurinos e três $(25 \%)$ em zebuínos. Informações obtidas e exames clínicos permitiram relacionar o problema à ação de ectoparasitas, como larvas de Dermatobia hominis, Tunga penetrans, infestações por Rhipicephalus microplus ou ausência de cuidados higiênicos, como a tosquia dos pelos prepuciais.

Dos cinco $(2,3 \%)$ reprodutores portadores de persistência de frênulo, todos apresentavam desvio ventral do pênis e incapacidade de cópula. Três (60\%) desses bovinos eram zebuínos e foram indicados, pelos respectivos proprietários, como portadores de baixa eficiência no teste de libido. Foi constatado no ato operatório, que um dos bovinos afetados por parafimose apresentou necrose da extremidade livre do pênis. Essa lesão foi confirmada pela histopatologia. 
Tabela 3: Frequência de enfermidades diagnosticadas na genitália externa de 217 touros, em 215 propriedades rurais do Estado de Goiás, no período de 2007 a 2013

\begin{tabular}{|c|c|c|c|}
\hline Local & Classificação & Quantidade & Frequência (\%) \\
\hline \multirow{8}{*}{ Prepúcio } & Abscesso & 12 & 7,10 \\
\hline & Acropostite-fimose & 136 & 80,49 \\
\hline & Dilaceração & 03 & 1,77 \\
\hline & Divertículo anterior & 01 & 0,59 \\
\hline & Divertículo posterior & 03 & 1,77 \\
\hline & Fimose & 09 & 5,32 \\
\hline & Frênulo persistente & 05 & 2,96 \\
\hline & Subtotal & 169 & 100 \\
\hline \multirow{6}{*}{ Pênis } & Desvio prematuro & 09 & 21,95 \\
\hline & Fibropapiloma de glande & 13 & 31,70 \\
\hline & Fratura & 11 & 26,83 \\
\hline & Parafimose & 04 & 9,76 \\
\hline & Urolitíase & 04 & 9,76 \\
\hline & Subtotal & 41 & 100 \\
\hline \multirow{5}{*}{$\begin{array}{l}\text { Bolsa escrotal e } \\
\text { testículos }\end{array}$} & Abscesso escrotal & 01 & 14,29 \\
\hline & Hérnia inguino-escrotal & 02 & 28,57 \\
\hline & Orquite & 03 & 42,85 \\
\hline & Seminoma testicular & 01 & 14,29 \\
\hline & Subtotal & 07 & 100 \\
\hline Total & & 217 & \\
\hline
\end{tabular}

Outra informação obtida na pesquisa revelou que, dos 215 produtores, 158 (73,50\%) efetuavam empiricamente o tratamento de eventuais enfermidades genitais dos touros, ignorando a importância da avaliação clínica dos animais. Por este motivo, diversos animais eram encaminhados ao abate, mesmo sendo de alto valor zootécnico, resultando em consideráveis prejuízos financeiros aos criatórios. Em muitos casos, a ausência ou o número insuficiente de médicos veterinários capacitados que atuam no campo foi mencionado como um dos principais pontos de estrangulamento e responsáveis pelas condutas acima mencionadas e não a falta de recursos financeiros dos criadores.

\section{Discussão}

O presente estudo demonstrou que $64 \%$ das enfermidades genitais externas de touros foram diagnosticadas na mesorregião Sul de Goiás. Esse fato foi atribuído à vasta extensão territorial dessa mesorregião em relação às demais e, também, a maior proximidade das propriedades com as duas instituições de ensino responsáveis pelo diagnóstico desses casos.

A maior ocorrência das enfermidades acometendo a genitália externa, com destaque para as lesões prepucias, como a acropostite-fimose nos touros zebuínos em relação aos taurinos, aqui evidenciada, 
pode estar relacionada a causas multifatoriais, em razão de suas particularidades anatômicas. Destacam-se o prepúcio e folheto prepucial interno pendulosos e o maior diâmetro do orifício prepucial, sendo essas características herdáveis e intrínsecas das raças zebuínas. Outros pesquisadores também fizeram referência a essas particularidades, responsabilizando tais aspectos, como fatores de risco para a ocorrência de enfermidades como acropostite-fimose e divertículo prepucial anterior $^{(1,2,4,15,16)}$. Os mesmos autores acrescentaram, ainda, que touros portadores de agenesia ou atrofia do músculo retrator do prepúcio podem apresentar óstio prepucial largo ou comprometimento da ação esfinctérica do óstio prepucial. Consequentemente, os touros apresentavam predisposição ao prolapso crônico do folheto prepucial interno, suscetibilizando-os aos traumas, com consequente perda da capacidade de serviço; portanto, não se pode desvincular a condição da gênese da acropostite-fimose, o que justificaria o maior número de casos registrados no presente trabalho.

Dados epidemiológicos, como ocorrência, frequência e incidência, bem como os fatores de risco quanto às diversas enfermidades acometendo a genitália externa de touros apresentados neste estudo, são raramente citados pela literatura especializada e apontam para a importância do acompanhamento técnico de um profissional qualificado no sistema de produção, tendo em vista o impacto econômico que essas afecções determinam. Estudos citados por Memon et al. ${ }^{(1)}$, Parker et al. ${ }^{(5)}$, e Kamiloglu et al. ${ }^{(17)}$ fazem referência a importância dos dados epidemiológicos de enfermidades no trato reprodutor de touros; todavia, retratam o problema em rebanhos de touros em outros países. No Brasil, exclusivamente no estado de Goiás, estudo retrospectivo (1982-2007) realizado por Rabelo et al. ${ }^{(6)}$ confirmou que de 12.320 touros, 898 eram portadores de enfermidades na genitália externa, enfatizando a importância do assunto e a necessidade de estudos complementares.

A etiopatogenia multifatorial, com destaque para a traumática, enfatizando as pastagens mal manejadas ou infestadas por plantas daninhas, bicadas de aves silvestres, com destaque para o carcará (Polyborus plancus), bem como a espoliação por ectoparasitas citados neste estudo, corrobora com as informações apresentadas por outros pesquisadores ${ }^{(3,4,18)}$. Estes achados encontraram sustentação nos trabalhos de Marques et al. ${ }^{(19)}$, Van Saun \& Ciszewski ${ }^{(20)}$ e Isa et al. ${ }^{(21)}$, os quais acrescentaram ainda a ausência de cuidados higiênico-sanitários do prepúcio dos touros como um fator importante na etiopatogenia das lesões genitais.

Os dados desta pesquisa apontaram maior ocorrência de lesões na genitália de touros com idade superior a 60 meses. Sabe-se que a idade dos animais está diretamente relacionada à dominância do rebanho, fato que contribui para que os touros mais velhos efetuem maior número de coitos, quando em comparação aos animais jovens do plantel. Sendo assim, estes animais mais velhos estão mais predispostos à lesões da genitália. Quadro semelhante foi descrito por Rabelo et al. ${ }^{(6)}$ Riet-Correa et al. ${ }^{(22)}$, que relataram que a prevalência das enfermidades presentes na genitália externa aumentou significativamente em reprodutores mais velhos.

A maior ocorrência de lesões presentes no prepúcio, pênis e testículos de touros em sistemas extensivos e ao término da estação de monta, quando esta era adotada, reflete a necessidade de cuidados sanitários e medidas de manejo dentro do sistema de produção de bovinos. Outros estudiosos $^{(4,16,17)}$, apesar de não especificarem o período de maior incidência de lesões na genitália de touros, enfatizaram a importância que a permanência direta ou mesmo as longas estações de monta têm sobre os maiores índices de observação de traumas ou acidentes ocorridos durante a cópula, culminando em enfermidades como a acropostite-fimose, abscessos prepuciais, fraturas penianas, dentre outras. 
A importância do conhecimento, por parte do profissional, das técnicas de bloqueios anestésicos locorregionais com dessensibilização dos nervos pudendos e hemorroidais é extremamente útil no auxílio do exame e realização do diagnóstico de algumas dessas enfermidades, com destaque para o desvio prematuro do pênis e fibropapiloma de glande ${ }^{(4,7,23)}$. Entretanto, em alguns casos, mesmo após o proprietário detectar a presença de subfertilidade ou infertilidade do touro e após exame clínico específico, houve dificuldade em se diagnosticar o tipo de enfermidade existente, sendo necessário empregar o teste de capacidade de serviço adotado por Santos et al. ${ }^{(8)}$. O método mostrou-se eficaz, uma vez que permitiu o diagnóstico de anormalidades na genitália de touros, às vezes indetectáveis ao exame clínico, como por exemplo, nos casos de desvio prematuro de pênis. O teste de comportamento sexual preconizado como conduta neste estudo mostrou-se efetivo no diagnóstico de várias enfermidades, com destaque ao desvio prematuro do pênis. Procedimento semelhante foi recomendado por Mcdiarmid ${ }^{(24)}$ como método de avaliação na elucidação de enfermidades penianas, principalmente nos casos de desvio prematuro e pênis infantil, justificando, portanto, seu emprego nesta investigação.

Os resultados deste estudo retrospectivo revelaram a acropostite-fimose, o fibropapiloma de glande e os abscessos prepuciais como as três enfermidades mais diagnosticas e reforçaram a importância da realização de estudos epidemiológicos regionais e nacionais, uma vez que essas informações podem servir de sustentação científica para outros estudos e podem auxiliar os profissionais veterinários da região no diagnóstico e controle dessas alterações. Estes achados diferem dos descritos por Kamiloglu et al. ${ }^{(20)}$, que registraram como principais lesões as fraturas penianas em 42 (28\%) bovinos acometidos, $29(19,30 \%)$ papilomas de glande, 22 (14,60\%) casos de acropostite-fimose e 22 $(14,60 \%)$ fimoses.

Os abscessos prepuciais diagnosticados neste estudo caracterizaram-se como uma importante enfermidade, principalmente nos touros taurinos. A relação entre a presença dos abscessos prepuciais e a espoliação por ectoparasitas apresentada na pesquisa, mostrou-se pertinente. A menor adaptação desses bovinos às condições de umidade e temperatura elevada, principalmente no Centro-Oeste brasileiro, propicia, indiscutivelmente, a infestação parasitária, fato este também descrito por Figueiredo et al. ${ }^{(25)}$.

Quanto ao fibropapiloma de glande, dados mencionados na anamnese apontaram uma correlação entre essa enfermidade e a forma cutânea da papilomatose bovina, sendo essa informação interpretada como uma importante informação epidemiológica. Rabelo et al. ${ }^{(6)}$, Gilbert et al. ${ }^{(26)}$, Monteiro et al. ${ }^{(27)}$ também enfatizaram essa possibilidade, ressaltando ainda que a sodomia, verificada especialmente em touros jovens, pode ocasionar lesão traumática na parte livre do pênis, em especial à glande, facilitando o contágio do epitélio peniano pelo agente.

Quanto à persistência de frênulo prepucial, este fato refletiu-se na característica de baixa libido apresentada por alguns touros, especialmente nas raças zebuínas. Acredita-se que estes animais, por ocasião da fase inicial da maturidade sexual, por apresentarem discreta libido, masturbam-se com menor frequência, o que em tese contribuiria para a persistência do frênulo prepucial após a puberdade. Essas informações corroboram as citações de Rabelo et al. ${ }^{(6)}$.

Os prejuízos econômicos, em virtude da presença das enfermidades acometendo a genitália externa de touros, apesar de não mensurados no estudo, foram considerados impactantes. A inexistência ou a escassez de profissionais que trabalham em serviços de extensão rural, considerado como um gargalo pelos pecuaristas, mereceu atenção dos pesquisadores, que, baseados na literatura ${ }^{(3,6)}$, consideravam o fator econômico e o valor intrínseco dos touros como principal causa do descarte prematuro ou 
mesmo do tratamento empírico muitas vezes verificado nas propriedades. Ressaltam-se também, como prejuízos econômicos em virtude das lesões acometendo a genitália externa de touros, os gastos com serviços profissionais, medicamentos e descarte prematuros de touros. Quadro semelhante foi discutido por Kamiloglu et al. ${ }^{(17)}$, que registraram, entre os anos de 2000 a 2003, 150 casos de desordens penianas e prepuciais diagnosticadas, dos quais aproximadamente 25 touros $(16,70 \%)$ foram descartados do plantel sem qualquer avaliação do quadro clínico do paciente ou tentativa de tratamento terapêutico e/ou cirúrgico, desconsiderando os prejuízos decorrentes das enfermidades.

\section{Conclusão}

Os resultados e informações apresentadas apontaram para a importância de estudos regionalizados dessa natureza, tendo em vista, principalmente, o entendimento da etiopatogenia, sua distribuição, fatores de risco e medidas preventivas relacionados às enfermidades que afetam a genitália externa de touros, bem como o impacto econômico regional dentro dos sistemas de produção.

\section{Referências}

1. Memom MA, Dawson LJ, Usenik EA, Rice LE. Preputial injuries in beef bulls: 172 cases (1980-1985). J. Am. Vet. Med. Assoc. 1988; 193:481-485.

2. Desrochers A, St-Jean G, Anderson DE. Surgical management of preputial injuries in bulls: 51 cases (19861994). Can. Vet. J. 1995; 36:553-556.

3. Rabelo RE, Silva LAF, Viu MAO, Romani AF, Alves CB., Fernandes JJR, Castro CFP. Acrobustite bovina: Revisão de literatura. Rev. CFMV. 2006; 37:29-36.

4. Rabelo RE, Silva OC. Aspectos morfofuncionais, clínicos e cirúrgicos do pênis, prepúcio e testículos de touros. Goiânia, Kelps, 2011. 212p.

5. Parker WG, Braun RK, Bean B, Hillman RB, Larson LL, Wilcox CJ. Avulsion of the bovine prepuce from its attachmentto the penile integument during semen collection with an artificial vagina. Theriogenology. 1987; 28:237-256.

6. Rabelo RE, Silva LAF, Brito LAB, Moura MI, Silva OC, Carvalho VS, Franco LG. Epidemiological aspects of surgical diseases of the genital tract in a population of 12,320 breeding bulls (1982-2007) in the state of Goias, Brazil. Ciên. Anim. Bras. 2008; 9:705-713.

7. Edmonson, MA. Local and regional anestesia in catlle. Veterinary clinics of North America: Food animal practice. 2008; 24: 211-226.

8. Santos MD, Torres CAA, Ruas JRM, Silva Filho JM, Costa EP, Pereira JC. Teste da libido e atividade de monta em touros da raça Nelore. Arq. Bras. Med. Vet. Zoot. 2004; 56:504-510.

9. Oliveira, CB, Guimarães, JD, Costa, EP, Siqueira, JB, Torres, CA, Carvalho, GR, Guimarães, SEF. Avaliação do comportamento sexual em touros Nelore: comparação entre os testes da libido em curral e do comportamento sexual a campo. Revista Brasileira de Zootecnia. 2007; 36 (1): 32-42.

10. Rabelo RE, Vulcani VAS, Cardoso LD, Dultra HT, Helrigel PA, Vincentin FR. Aspectos anatômicos e sua relação com as enfermidades do prepúcio e pênis do touro. Rev. Eletri. Med. Vet. 2012;9(18):1-24.

11. Musser, JMB, St Jean, G, Westweber, AG, Pejsa, TG. Penile hematoma in bulls: 60 cases (1979-1990). Journal of the American Veterinary Medical Association. 1992; 201 (9): 416-418. 
12. Eurides D, Silva M, Coelho HE, Contesini EA. Implante de retalho de silicone em pênis de bovinos. Estudo experimental. Ciência Rural. 1994;24(3):545-550.

13. Thrusfield M, Mansley L, Dunlop P, Taylor J, Pawson A, Stringer L. The foot-and-mouth disease epidemic in Dumfries and Galloway: Characteristics and control. Vet. Rec. 2005; 8:229-252.

14. Sampaio IBM. Estatística Aplicada à Experimentação Animal. $2^{\mathrm{a}}$ ed., Belo Horizonte: Fundação de Ensino e Pesquisa em Medicina Veterinária e Zootecnia, 2002. 265p.

15. Viu MAO, Tonhati H, Cerón-Munõz MF. Parâmetros genéticos do peso e escores visuais de prepúcio e umbigo em gado de corte. ARS Veterinária. 2002; 2:179-184.

16. Ashdown RR. Functional, developmental and clinical anatomy of the bovine pênis and prepuce. CAB Reviews: Perspectives in agriculture, Veterinary Science, Nutrion and Natural Resources, London, 2006; 1(21):1-29.

17. Kamiloglu A, Ozturk S, Klc E, Aksoy O. Management of penile and preputial disorders in bulls: 150 cases (200-2003), Kafkas Univ Vet Fak Derg. 2004; 1:31-36. http://www.cabaabstractsplus.org/google/abstract.asp?AcNo=20053003830. Acesso 12/01/2014.

18. Copland, RS, Baker, AA, Rival, MD, Lennon, PE. Treatment of injuries on the penis and prepuce of bulls. Australian Veterinary Journal. 1989; 66 (11): 378-380.

19. Marques JA, Marques LC, Canola JC, Cattelan JW. A acropostite-fimose em touros - uma técnica cirúrgica de tratamento. Ciênc. Vet. 1988.; 2:2-3.

20. Van Saun RJ, Ciszewski DK. Preputial foreign body in a bull. J. Am. Vet. Med. Assoc. 1989; 9:1303-1304.

21. Isa JF, Magembe SR, Naude TW. Devil's claw (Harpagophytum procumbens) in a Brahman's preputial sheath: a case report from Botswana. J. South Afric. Vet. Assoc. 2001; 72:55-56.

22. Riet-Correa F, Freitas A, Puignau MV. Ulcerative posthitis in bulls in Uruguay. Cornell Vet. 1979; 69:3344.

23. Wolfe DF, Hudson RS, Walker DF. Common penile and preputial problems of bulls. Compend. Contin. Educ. Pract. Vet. 1983;5 (8):447-452.

24. Mcdiarmid JJ. "Corkscrew penis" and other breeding abnormalities in beef bulls. N. Z. Vet. J. 1981; 29:3536.

25. Figueiredo LTM, Badra SJ, Pereira LE. Relato sobre carrapatos coletados no sudeste e centro-oeste do Brasil, analisando a potencial transmissão de microorganismos de carrapatos para o homem. Rev. Soc. Bras. Med. Trop. 1999; 32:613-619.

26. Gilbert, RO, Lindsay, WA, Levine, SA. Successful surgical repair of a vascular shunt of the corpus cavernosum penis and penile fibropapiloma in a bull. Journal South African Veterinary Association. 1987; 58 (4): 193-195.

27. Monteiro, VLC, Coelho, MCOC, Carneiro, AS, Silva, RA, Teixeira, MN, Wanderley, AG, Wanderley, EK, Franco, ES. Descrição clínica e histopatológica da papilomatose cutânea bovina. Ciênc. Anim. Bras. 2008; 9 (4): 1079-1088. 